\title{
On the postulation of lines and a fat line
}

Thomas Bauer, Sandra Di Rocco, David Schmitz, Tomasz Szemberg, Justyna Szpond

October 11, 2018

\begin{abstract}
In the present note we show that the union of $r$ general lines and one fat line in $\mathbb{P}^{3}$ imposes independent conditions on forms of sufficiently high degree $d$, where the bound is independent of the number of lines. This extends former results of Hartshorne and Hirschowitz on unions of general lines, and of Aladpoosh on unions of general lines and one double line.
\end{abstract}

Keywords postulation problems, fat flats, Hilbert functions, Serre vanishing

Mathematics Subject Classification (2010) 14C20, 14F17, 13D40, 14N05

\section{Introduction}

Let $X \subset \mathbb{P}^{n}$ be a closed subscheme. The Hilbert function of $X$ encodes a number of properties of $X$ and has been classically an object of vivid research in algebraic geometry and commutative algebra. We recall first the definition.

Definition 1.1 (Hilbert function). The Hilbert function of a scheme $X \subset \mathbb{P}^{n}(\mathbb{K})$ is

$$
\operatorname{HF}_{X}: \mathbb{Z} \ni d \rightarrow \operatorname{dim}_{\mathbb{K}}[S(X)]_{d} \in \mathbb{Z}
$$

where $S(X)$ denotes the graded homogeneous coordinate ring of $X$.

It is well known that the Hilbert function becomes eventually (i.e., for large $d$ ) a polynomial. We denote this Hilbert polynomial of $X$ by $\mathrm{HP}_{X}$. Whereas the Hilbert polynomial can be (in principle) computed algorithmically, the Hilbert function is more difficult to compute. It may happen that the Hilbert function is equal to the Hilbert polynomial, for example for $\mathbb{P}^{n}$ we have $\operatorname{HF}_{\mathbb{P}^{n}}(d)=\operatorname{HP}_{\mathbb{P}^{n}}(d)$ for all $d \geqslant 0$, but this behaviour is rare. The next simplest behaviour occurs for subschemes with bipolynomial Hilbert function.

Definition 1.2 (Bipolynomial Hilbert function). Following [4] we say that $X$ has a bipolynomial Hilbert function if

$$
\operatorname{HF}_{X}(d)=\min \left\{\operatorname{HP}_{\mathbb{P}^{n}}(d), \operatorname{HP}_{X}(d)\right\}
$$

for all $d \geqslant 1$.

In other words, $X$ has a bipolynomial Hilbert function if $X \subset \mathbb{P}^{n}$ imposes the expected number of conditions on forms of arbitrary degree $d \geqslant 1$. It is definitional that if $X$ consists of $q$ general points in $\mathbb{P}^{n}$, then its Hilbert function is bipolynomial. An analogous result for $X$ consisting of $r$ general lines in $\mathbb{P}^{n}$ with $n \geqslant 3$ has 
been proved by Hartshorne and Hirschowitz in [9, Theorem 0.1]. Recently Carlini, Catalisano and Geramita [6] showed that if $X$ consists of $r$ general lines and one general fat point, then, up to a short list of exceptions in $\mathbb{P}^{3}$, the Hilbert function of $X$ is bipolynomial, see also [2] and [3].

Aladpoosh in [1] has proved recently that also a scheme consisting of $r$ general lines and one double line has (with the exception of one double line and two simple lines in $\mathbb{P}^{4}$ imposing dependent conditions on forms of degree 2) a bipolynomial Hilbert function. She also conjectured [1, Conjecture 1.2] that the same holds true for $r$ general lines and one fat flat of arbitrary dimension. In the present note we provide evidence supporting this conjecture for a fat line of arbitrary multiplicity $m$. Our main result is the following.

Main Theorem. Let $m \geqslant 1$ be a fixed integer. Then for $d \geqslant d_{0}(m):=3\left(\begin{array}{c}m+1 \\ 3\end{array}\right)$, the Hilbert function of a subscheme $X \subset \mathbb{P}^{3}$ consisting of $r \geqslant 0$ general lines and one line of multiplicity $m$ (i.e. defined by the $m$-th power of the ideal of a line) satisfies formula (1).

In other words, a general fat line and an arbitrary number $r$ of general lines with multiplicity 1 impose independent conditions on forms of degree $d \geqslant d_{0}(m)$ (see Theorem 4.1).

It follows from the Serre Vanishing [10, Theorem 1.2.6] that for any subscheme $X \subset \mathbb{P}^{n}$, there exists a bound $d_{0}(X)$ such that $X$ imposes independent conditions on forms of degree $d \geqslant d_{0}(X)$. The point here is that we obtain an explicit bound that depends only on the multiplicity of the fat line but is independent of the number of reduced lines.

We will set up the proof in a way which employs the general strategy of Hartshorne and Hirschowitz [9] and Carlini, Catalisano and Geramita 6]. This amounts to work inductively by constructing a a suitable sequence of generic subschemes $Z_{0}, Z_{1}, \ldots$, along with suitable specializations $Z_{i}^{\prime}$ of $Z_{i}$. The starting scheme $Z_{0}$ consists of the lines in the theorem plus a number of generic points. The essential difficulty in this strategy lies in the question which kinds of intermediate schemes $Z_{i}$ to consider and which specializations $Z_{i}^{\prime}$ to chose, in order for an inductive procedure to work. In our approach this is achieved by using intermediate schemes that contain, apart from disjoint lines and points, also crosses and so-called zig-zags (see Def. 2.3).

\section{Preliminaries and auxiliary results}

We begin by recalling a formula for the number $c(n, m, d)$ of conditions which vanishing to order $m$ along a line in $\mathbb{P}^{n}$ imposes on forms of degree $d \geqslant m$ :

$$
c(n, m, d)=\frac{m(n d+2 n+m-m n-1)}{n(n-1)}\left(\begin{array}{c}
n+m-2 \\
m
\end{array}\right) .
$$

For a proof see e.g. [7, Lemma 2.1]. Note that

$$
c(n, 1, d)=d+1
$$

for all $n \geqslant 1$.

In the next Lemma we present a useful formula relating some of numbers $c(n, m, d)$.

Lemma 2.1. For all positive integers $n, m, d$ we have

$$
c(n, m, d)=c(n, m-1, d-1)+c(n-1, m, d) .
$$


Proof. This is a straightforward computation.

In $\mathbb{P}^{3}$ the formula (2) reduces to

$$
c(d, m)=c(3, m, d)=\frac{1}{6} m(m+1)(3 d+5-2 m) .
$$

Our approach to the Main Theorem uses the specialization method. This employs the Semi-Continuity Theorem [8, Theorem III.12.8] in the following way:

Let $f: X \rightarrow B$ be a projective morphism of noetherian schemes and let $\mathcal{F}$ be a coherent sheaf on $X$, flat over $B$. The vanishing $h^{0}\left(X_{b}, \mathcal{F}_{b}\right)=0$ for some $b$ implies then the vanishing $h^{0}\left(X_{b^{\prime}}, \mathcal{F}_{b^{\prime}}\right)=0$ for all $b^{\prime}$ in a neighborhood of $b$.

In our situation, this means concretely that if $h^{0}\left(\mathbb{P}^{n}, \mathcal{O}_{\mathbb{P}^{n}}(d) \otimes \mathcal{J}_{Z_{b}}\right)=0$ for a (special) subscheme $Z_{b}$, then $h^{0}\left(\mathbb{P}^{n}, \mathcal{O}_{\mathbb{P}^{n}}(d) \otimes \mathcal{J}_{Z_{b^{\prime}}}\right)=0$ for a (general) subscheme $Z_{b^{\prime}}$ such that $Z_{b}$ and $Z_{b^{\prime}}$ vary in a flat family over $B$.

We are going to use and generalize the notion of sundials following the ideas of Carlini, Catalisano and Geramita, see [6, Section 2] for definitions and motivations.

Definition 2.2 (Sundials and crosses). A sundial in $\mathbb{P}^{n}$ is the limiting subscheme obtained by a collision of two skew lines (hence spanning a $\mathbb{P}^{3} \subset \mathbb{P}^{n}$ ). It has a nonreduced structure in the collision point which can be thought of as a vector generating together with the plane spanned by the two intersecting lines the $\mathbb{P}^{3}$ mentioned above.

A union of two lines in $\mathbb{P}^{n}$ intersecting in a single point is called a cross. A cross is hence a sundial with the reduced structure.

Carlini, Catalisano and Geramita proved in [4, Lemma 2.5] that there exists a flat family $g: W \rightarrow B$ of schemes in $\mathbb{P}^{n}$, with $n \geqslant 3$ such that a general member $W_{b^{\prime}} \subset W$ is a union of two disjoint lines, whereas the special fiber $W_{b}$ is a sundial.

It is a crucial point in our proof of the Main Theorem to use a generalization of this idea, which uses zig-zags in the following sense:

Definition 2.3 (Zig-zag). A zig-zag of length $z$ is the limiting subscheme obtained by a collision of an ordered set of $z$ general lines $L_{1}, L_{2}, \ldots, L_{z}$ in such a way, that the line $L_{1}$ intersects $L_{2}$, the line $L_{2}$ intersects $L_{1}$ and $L_{3}$ and the intersection points are distinct, $L_{3}$ intersects $L_{2}$ and $L_{4}$ and the intersection points are again distinct, and so on, finally $L_{z-1}$ intersects $L_{z-2}$ and $L_{z}$ in two distinct points. The structure in the intersection points is the same as the structure of a sundial in the intersection point of its lines. A zig-zag of length $z$ has thus $(z-1)$ singular points.

A reduced zig-zag is a zig-zag with reduced structure, i.e., no embedded points.

Figure 1 shows a zig-zag of length 7 . Note that the lines in the figure are all skew, there are no other intersection points but those indicated in this figure. The intersection points are embedded points with the structure of a scheme of length 2 not contained in the plane generated by the intersecting lines. Note that a sundial is just a zig-zag of length 2 . A cross is a reduced zig-zag of length 2 .

Lemma 2.4. For an integer $z \geqslant 2$, there exists a flat family $\left\{X_{\lambda}\right\}$ of schemes in $\mathbb{P}^{n}$, with $n \geqslant 3$ such that a general member of $\left\{X_{\lambda}\right\}$ is a union of $z$ disjoint lines and the special fiber is a zig-zag of length $z$.

Proof. The proof consists in a generalization of the argument in [4, Lemma 2.5]. 


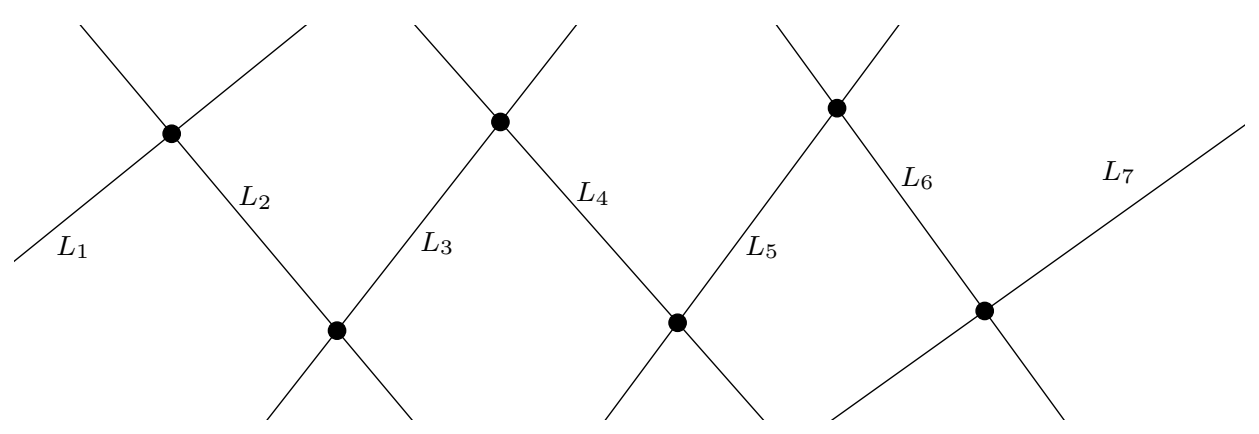

Figure 1: A zig-zag of length 7

Zig-zags are useful in our approach because of the following fact.

Lemma 2.5. Let $S$ be a zig-zag of length $z$ in $\mathbb{P}^{3}$ formed by lines $L_{1}, \ldots, L_{z}$. Let $Q$ be a smooth quadric in $\mathbb{P}^{3}$ such that all singular points of $S$ lie on $Q$ but none of the lines in $S$ is contained in $Q$. Then the colon ideal

$$
J=I_{S}: I_{Q}
$$

defines the reduced zig-zag $V(J)=L_{1} \cup \ldots \cup L_{z}$.

Apart from semicontinuity, the residual exact sequence and the Castelnuovo inequality are key ingredients in the proof. We discuss them now.

Definition 2.6 (Trace and residual scheme). Let $Y$ be a divisor of degree $e$ in $\mathbb{P}^{n}$ and let $Z \subset \mathbb{P}^{n}$ be a closed subscheme. Then the subscheme $Z^{\prime \prime}=\operatorname{Tr}_{Y}(Z)$ defined in $Y$ by the ideal

$$
I_{Z^{\prime \prime} / Y}=\left(I_{Y}+I_{Z}\right) / I_{Y} \subset \mathcal{O}_{Y}
$$

is the trace of $Z$ on $Y$.

The colon ideal $I_{Z^{\prime}}=\left(I_{Z}: I_{Y}\right) \subset \mathcal{O}_{\mathbb{P}^{n}}$ defines $Z^{\prime}=\operatorname{Res}_{Y}(Z)$, the residual scheme of $Z$ with respect to $Y$.

One has the following residual exact sequence

$$
0 \longrightarrow \mathcal{J}_{Z^{\prime}}(-Y) \longrightarrow \mathcal{J}_{Z} \longrightarrow \mathcal{J}_{Z^{\prime \prime} / Y} \longrightarrow 0
$$

where $\mathcal{J}_{W}$ is the sheafification of the ideal $I_{W}$. Twisting (3) by $\mathcal{O}_{\mathbb{P} n}(d)$ we get

$$
0 \longrightarrow \mathcal{O}_{\mathbb{P}}(d-e) \otimes \mathcal{J}_{\operatorname{Res}_{Y}(Z)} \longrightarrow \mathcal{O}_{\mathbb{P}^{n}}(d) \otimes \mathcal{J}_{Z} \longrightarrow \mathcal{O}_{Y}(d) \otimes \mathcal{J}_{\operatorname{Tr}_{Y}(Z)} \longrightarrow 0 .
$$

Taking then the long cohomology sequence of (4) we obtain the following statement, which is called the Castelnuovo inequality, see e.g. [5, Lemma 3.3].

Lemma 2.7 (Castelnuovo inequality). Let $Y \subset \mathbb{P}^{n}$ be a divisor of degree $e$ and let $d \geqslant e$ be an integer. Let $Z \subset \mathbb{P}^{n}$ be a closed subscheme. Then

$$
h^{0}\left(\mathbb{P}^{n}, \mathcal{O}_{\mathbb{P}^{n}}(d) \otimes \mathcal{J}_{Z}\right) \leqslant h^{0}\left(\mathbb{P}^{n}, \mathcal{O}_{\mathbb{P}^{n}}(d-e) \otimes \mathcal{J}_{\operatorname{Res}_{Y}(Z)}\right)+h^{0}\left(Y, \mathcal{O}_{Y}(d) \otimes \mathcal{J}_{\operatorname{Tr}_{Y}(Z) / Y}\right)
$$

We call the space $H^{0}\left(\mathbb{P}^{n}, \mathcal{O}_{\mathbb{P}^{n}}(d-e) \otimes \mathcal{J}_{\operatorname{Res}_{Y}(Z)}\right)$ the residual linear system of $H^{0}\left(\mathbb{P}^{n}, \mathcal{O}_{\mathbb{P}^{n}}(d) \otimes \mathcal{J}_{Z}\right)$ with respect to $Y$ and $H^{0}\left(Y, \mathcal{O}_{Y}(d) \otimes \mathcal{J}_{\operatorname{Tr}_{Y}(Z) / Y}\right)$ the trace linear system of $H^{0}\left(\mathbb{P}^{n}, \mathcal{O}_{\mathbb{P}^{n}}(d) \otimes \mathcal{J}_{Z}\right)$ on $Y$. 


\section{Nonspeciality of certain linear series on $\mathbb{P}^{1} \times \mathbb{P}^{1}$}

In the proof of the Main Theorem we will consider trace linear systems on a smooth quadric in $\mathbb{P}^{3}$. This section serves as a preparation of relevant results on linear systems on a smooth quadric in $\mathbb{P}^{3}$ identified with $\mathbb{P}^{1} \times \mathbb{P}^{1}$. Special linear systems with general points of multiplicity at most 3 on $\mathbb{P}^{1} \times \mathbb{P}^{1}$ have been classified by Lenarcik in [11]. Here we recall a part of [11, Theorem 2] relevant in our situation.

Lemma 3.1. Let $Z$ be the fat point scheme in $\mathbb{P}^{1} \times \mathbb{P}^{1}$ defined by the ideal

$$
I_{Z}=I\left(P_{1}\right)^{2} \cap \ldots \cap I\left(P_{p}\right)^{2} \cap I\left(Q_{1}\right) \cap \ldots \cap I\left(Q_{q}\right),
$$

where $P_{1}, \ldots, P_{p}, Q_{1}, \ldots, Q_{q}$ are general points in $\mathbb{P}^{1} \times \mathbb{P}^{1}$. Let $0 \leqslant a \leqslant b$ be nonnegative integers. The linear system

$$
H^{0}\left(\mathbb{P}^{1} \times \mathbb{P}^{1}, \mathcal{O}_{\mathbb{P}^{1} \times \mathbb{P}^{1}}(a, b) \otimes \mathcal{J}_{Z}\right)
$$

is special if and only if one of the following cases holds

- $a=0$ and $p+2 q \leqslant b$,

- $a=2, p=0, b=q-1$ and $q$ is odd.

Using this result, we prove now an auxiliary postulation statement for higher multiplicities:

Lemma 3.2. Given an integer $m \geqslant 2$ let $k$ be an integer with $k \geqslant\left(\begin{array}{c}m+1 \\ 3\end{array}\right)$. Then 2 general points $P_{1}, P_{2}$ taken with multiplicity $m$ impose independent conditions on linear systems on $\mathbb{P}^{1} \times \mathbb{P}^{1}$ of bidegree $(a, b)$ if $a \leqslant b$ and $a \geqslant k-1$ and $b \geqslant 3 k$.

Proof. For $m=2$ the assertion for arbitrary $k \geqslant\left(\begin{array}{c}m+1 \\ 3\end{array}\right)$ follows from Lemma 3.1. We proceed by induction on $m$ and $k$. Let $m$ and $k \geqslant\left(\begin{array}{c}m+1 \\ 3\end{array}\right)$ be fixed and assume that the assertion holds for all $m^{\prime}<m$ and $k^{\prime}$. Let $s=(a+1)(b+1)-2\left(\begin{array}{c}m+1 \\ 2\end{array}\right)$ and let $Q_{1}, \ldots, Q_{s}$ be $s$ general points in $\mathbb{P}^{1} \times \mathbb{P}^{1}$. It is enough to show that there is no divisor of bidegree $(a, b)$ which passes with multiplicity $m$ through the points $P_{1}, P_{2}$ and passes through $Q_{1}, \ldots, Q_{s}$. It suffices to prove this claim for a particular position of points $Q_{1}, \ldots, Q_{s}$.

To this end let $C$ be a smooth curve of bidegree $(1,1)$ passing through $P_{1}$ and $P_{2}$. Thus $C$ is a smooth rational curve. Let $t=a+b-2 m+1$. By above assumptions this is a non-negative integer. We specialize now the points $Q_{1}, \ldots, Q_{t}$ onto the curve $C$ leaving the points $Q_{t+1}, \ldots, Q_{s}$ as general points on $\mathbb{P}^{1} \times \mathbb{P}^{1}$, so that they do not lie on $C$ in particular. Assume to the contrary that there is a divisor $\Gamma$ such that $\operatorname{mult}_{P_{i}} \Gamma \geqslant m$ for $i=1,2$ and $\operatorname{mult}_{Q_{j}} \Gamma \geqslant 1$ for $j=1, \ldots, s$. Then $C$ must be a component of $\Gamma$, because $(\Gamma \cdot C)=a+b$ but the trace of $\Gamma$ on $C$ has at least 2 points of multiplicity $m$ and another $t$ points with $2 m+t=a+b+1$. The residual divisor $\Gamma^{\prime}=\Gamma-C$ has bidegree $(a-1, b-1)$ and passes through the points $P_{1}$ and $P_{2}$ with multiplicity $m-1$ and also passes through the points $Q_{t+1}, \ldots, Q_{s}$. Since $s-t=a b-2\left(\begin{array}{c}m \\ 2\end{array}\right)$, the existence of $\Gamma^{\prime}$ is excluded by our induction assumption.

Thus we are done with the proof of the Lemma. 


\section{The proof of the Main Theorem}

In this section we will prove the Main Theorem, which is equivalent to the following statement.

Theorem 4.1 (Maximal rank property). For a subscheme $W \subset \mathbb{P}^{n}$ consisting of a general line of multiplicity $m$ and an arbitrary number $r$ of general lines, for all $d \geqslant d_{0}(m)=3\left(\begin{array}{c}m+1 \\ 3\end{array}\right)$, the restriction map

$$
H^{0}\left(\mathbb{P}^{n}, \mathcal{O}_{\mathbb{P}^{n}}(d)\right) \rightarrow H^{0}\left(W, \mathcal{O}_{W}(d)\right)
$$

has maximal rank.

As pointed out in the introduction, we will employ the general strategy of Hartshorne and Hirschowitz [9, Theorem 1.1]. Specifically, we will proceed inductively along a suitable sequence of subschemes $Z_{0}, Z_{1}, \ldots$, for which we choose suitable specializations $Z_{0}^{\prime}, Z_{1}^{\prime}, \ldots$ While we can start with a subscheme $Z_{0}$ consisting of general lines, a fat line and points, it is a major obstacle that it seems insufficient to use only these kinds of schemes during the whole induction process. Our idea is to instead allow intermediate schemes $Z=Z(m, r, s, q, z)$ consisting of one general line of multiplicity $m, r$ general lines, $s$ general crosses, $q$ general points and a reduced zig-zag of length $z$ (along with particular specializations $Z^{\prime}$ of $Z$, which will be introduced in Definition 4.4).

We now set up some notation that will be useful for the remainder of the paper. We denote by

$$
\mathcal{L}(k, \varepsilon ; m, r, s, q, z)=\mathcal{L}(d ; Z)=H^{0}\left(\mathbb{P}^{3}, \mathcal{O}_{\mathbb{P}^{3}}(d) \otimes \mathcal{J}_{Z}\right)
$$

the linear system of polynomials in $\mathbb{P}^{3}$ of degree $d=3 k+\varepsilon$, with $\varepsilon \in\{0,1,2\}$ vanishing along the subscheme $Z$.

Similarly we will write

$$
\Lambda\left((a, b) ; p, p_{d}, p_{m}, m\right)=\Lambda((a, b) ; \Omega)=H^{0}\left(\mathbb{P}^{1} \times \mathbb{P}^{1}, \mathcal{O}_{\mathbb{P}^{1} \times \mathbb{P}^{1}}(a, b) \otimes \mathcal{J}_{\Omega}\right)
$$

to indicate the linear system on $\mathbb{P}^{1} \times \mathbb{P}^{1}$ of polynomials of bidegree $(a, b)$ vanishing along the subscheme $\Omega=\Omega\left(p, p_{d}, p_{m}, m\right)$ consisting of $p$ general points, $p_{d}$ general double points and $p_{m}$ general points of multiplicity $m$. In our considerations $p_{m}$ is either 0 or 2 , depending on whether we specialize the fat line onto the quadric or not.

Given $m \geqslant 1$ and $d \geqslant d_{0}(m)=3\left(\begin{array}{c}m+1 \\ 3\end{array}\right)$ there exist unique integers $r(d, m) \geqslant 0$ and $0 \leqslant q(d, m) \leqslant d$ such that

$$
\operatorname{HP}_{\mathbb{P}^{3}}(d)=c(d, m)+r(d, m)(d+1)+q(d, m) .
$$

So $\operatorname{HP}_{\mathbb{P}^{3}}(d)$ is the virtual number of conditions that one $m$-fold line, $r(d, m)$ generic ordinary lines, and $q(d, m)$ generic points impose.

Remark 4.2. Concretely, we have

$$
r(d, m)=\left\lfloor\frac{1}{d+1}\left(\left(\begin{array}{c}
d+3 \\
3
\end{array}\right)-\frac{1}{6} m(m+1)(3 d+5-2 m)\right)\right\rfloor
$$

and

$$
q(d, m)=\left(\begin{array}{c}
d+3 \\
3
\end{array}\right)-\frac{1}{6} m(m+1)(3 d+5-2 m)-(d+1) r(d, m) .
$$

In particular, 
- for $d=3 k$

$$
r(d, m)=\frac{3}{2} k^{2}+\frac{5}{2} k+1-\left(\begin{array}{c}
m+1 \\
2
\end{array}\right) \text { and } q(d, m)=2\left(\begin{array}{c}
m+1 \\
3
\end{array}\right),
$$

- for $d=3 k+1$

$$
r(d, m)=\frac{3}{2} k^{2}+\frac{7}{2} k+2-\left(\begin{array}{c}
m+1 \\
2
\end{array}\right) \text { and } q(d, m)=2\left(\begin{array}{c}
m+1 \\
3
\end{array}\right),
$$

- for $d=3 k+2$

$$
r(d, m)=\frac{3}{2} k^{2}+\frac{9}{2} k+3-\left(\begin{array}{c}
m+1 \\
2
\end{array}\right) \text { and } q(d, m)=k+1+2\left(\begin{array}{c}
m+1 \\
3
\end{array}\right) .
$$

The following theorem (to be proved in Subsection 4.1) implies the Main Theorem.

Theorem 4.3. Let $d \geqslant d_{0}(m)=3\left(\begin{array}{c}m+1 \\ 3\end{array}\right)$ and let $Z=Z(m, r(m, d), 0, q(m, d), 0)$, or $Z=Z(m, r(m, d)+1,0,0,0)$. Let further be $Q$ some smooth quadric. Then there exists a sequence $Z=Z_{0}, Z_{1}, \ldots, Z_{u}$ of schemes $Z_{i}=Z\left(m_{i}, r_{i}, s_{i}, q_{i}, z_{i}\right)$ together with specializations $Z_{i}^{\prime}$ such that the following hold for each $i=0, \ldots, u-1$

(1) $Z_{i+1}=\operatorname{Res}_{Q}\left(Z_{i}^{\prime}\right)$

(2) $h^{0}\left(Q, \mathcal{O}_{Q}(d-2 i) \otimes I_{T r_{Q}\left(Z_{i}^{\prime}\right)}\right)=0$

and such that $Z_{u}$ satisfies the conditions

(i) $Z_{u}=Z\left(m_{u}, r\left(m_{u}, d-2 u\right), 0, q\left(m_{u}, d-2 u\right), 0\right)$, or $Z_{u}=Z\left(m_{u}, r\left(m_{u}, d-2 u\right)+1,0,0,0\right)$

(ii) $d-2 u \geqslant d_{0}\left(m_{u}\right)$

(iii) $m_{u} \in\{m-1, m-2,1,0\}$

Proof of Theorem 4.1. We proceed by induction on $m$. The base case $m=1$ has been proved for all $d \geqslant 0=d_{0}(1)$ in [9] and the base case $m=2$ by Aladpoosh [1] for all $d \geqslant 2=d_{0}(2)$.

Let now $m \geqslant 3$. For $d \geqslant d_{0}(m)$ it suffices to prove the bijectivity of the restriction map in the case of schemes $Z=Z(m, r(d, m), 0, q(d, m), 0)$, and the injectivity in the case of schemes $Z=Z(m, r(m, d)+1,0,0,0)$. This amounts in either case to proving the identity

$$
h^{0}(\mathcal{L}(d ; Z))=0 .
$$

Now, Theorem 4.3 together with Castelnuovo's inequality yields

$$
\begin{aligned}
h^{0}(\mathcal{L}(d ; Z)) & \leqslant h^{0}\left(\mathcal{L}\left(d-2 u ; Z_{u}\right)\right)+\sum_{i=1}^{u-1} h^{0}\left(Q, \mathcal{O}_{Q}(d-2 i) \otimes I_{\operatorname{Tr}_{Q}\left(Z_{i}^{\prime}\right)}\right) \\
& =h^{0}\left(\mathcal{L}\left(d-2 u ; Z_{u}\right)\right),
\end{aligned}
$$

but the latter must be zero since $Z_{n}$ satisfies the induction hypothesis, again by Theorem 4.3 . 


\subsection{Proof of Theorem 4.3}

In order to prove Theorem 4.3, we will need the next lemma describing which schemes result from certain specializations.

Definition 4.4. Let $Q$ be a smooth quadric in $\mathbb{P}^{3}$. We denote by $R\left(\delta, \ell, \ell_{s}, \ell_{z}, t, t_{s}, t_{z}\right)$ the specialization $Z^{\prime}$ of $Z=Z(m, r, s, q, z)$ given by assuming the following lines to be disjoint lines belonging to the same ruling of $Q$ :

- $\delta m$-fold lines (here $\delta$ will be either 0 or 1 );

- $\ell$ ordinary lines;

- $\ell_{s}$ lines from $\ell_{s}$ crosses (one line from each cross);

- $\ell_{z}=\left\lfloor\frac{z}{2}\right\rfloor$ lines from the reduced zig-zag of length $z$,

and assuming furthermore

- $t$ among the $q$ points to be general points on $Q$,

- $2 t_{s}$ of the $r$ lines to form $t_{s}$ sundials whose intersection with $Q$ is a zerodimensional scheme containing the singular points of the sundials,

- $t_{z}+1$ of the lines to form one zig-zag whose zero-dimensional intersection with $Q$ contains all $t_{z}$ singular points.

Lemma 4.5. Let $Z^{\prime}$ be the specialization $R\left(\delta, \ell, \ell_{s}, \ell_{z}, t, t_{s}, t_{z}\right)$ of the scheme $Z=$ $Z(m, r, s, q, z)$. Then

$\operatorname{Res}_{Q}\left(Z^{\prime}\right)=Z\left(m-\delta, r-\ell+\ell_{s}+\left(z-\ell_{z}\right)-2 t_{s}-\left(t_{z}+1\right), s-\ell_{s}+t_{s}, q-t, t_{z}+1\right)$

and

$\operatorname{Tr}_{Q}\left(Z^{\prime}\right)=D+\Omega\left(2 r-2 \ell-2 \ell_{z}-3 \ell_{s}-2 t_{s}-2 t_{z}+t+4 s+z+\gamma, t_{s}+t_{z}, 2-2 \delta, m\right)$,

where $D$ is a divisor on $Q$ consisting of $\delta$ lines, where $\delta \in\{0,1\}$, of multiplicity $m$ and $\ell+\ell_{s}+\ell_{z}$ reduced lines, all contained in the same ruling on $\mathbb{Q}$. Here $\gamma=$ $\left\{\begin{array}{ll}0, & \text { if } \ell_{z}=0 \\ 1, & \text { if } \ell_{z}>0\end{array}\right.$.

Now we turn to the proof of Theorem 4.3 ,

The particular sequence of subschemes differs according to the divisibility of $d$ by 3 . In order to simplify notation we denote the relevant linear series by

$$
\begin{aligned}
B(k, \varepsilon, m) & =\mathcal{L}(k, \varepsilon ; m, r(3 k+\varepsilon, m), 0, q(3 k+\varepsilon, m), 0) \\
I(k, \varepsilon, m) & =\mathcal{L}(k, \varepsilon ; m, r(3 k+\varepsilon, m)+1,0,0,0)
\end{aligned}
$$

The following table shows for each case the length and the final element of the sequence that we will construct in the sequel. 


\begin{tabular}{ccc}
\hline For & a sequence of length & yields \\
\hline$B(k, 0, m)$ & 1 & $B(k-1,1, m-1)$ \\
$B(k, 1, m)$ & 2 & $B(k-1,0, m-1)$ \\
$B(k, 2, m)$ & 1 & $B(k, 0, m-1)$ \\
\hline$I(k, 0, m)$ & 2 & $I(k-2,2, m-2)$ \\
$I(k, 1, m)$ & 1 & $I(k-1,2, m-1)$ \\
$I(k, 2,3 \ell)$ & $3 \ell-1$ & $B(k-2 \ell+1,1,1)$ \\
$I(k, 2,3 \ell+1)$ & $3 \ell+1$ & $B(k-2 \ell, 0,0)$ \\
$I(k, 2,3 \ell+2)$ & $3 \ell+1$ & $B(k-2 \ell, 0,1)$ \\
\hline
\end{tabular}

\subsubsection{The bijective cases}

With $d=3 k+\varepsilon$, the initial system in every case here is

$$
\mathcal{L}(k, \varepsilon ; m, r(3 k+\varepsilon, m), 0, q(3 k+\varepsilon, m), 0) .
$$

Case $B(k, 0, m)$. We only specialize once, and we pick

$$
Z_{0}^{\prime}=R(1,2 k+1-m, 0,0, m(m-1), 0,0) .
$$

By Lemma 4.5, we obtain the trace system

$H^{0}\left(\mathcal{O}_{Q}(d) \otimes I_{\operatorname{Tr}_{Q}\left(Z^{\prime}\right)}\right)=\Lambda((d, d-(2 k+1)) ; 2 r-2(2 k+1-m)+m(m-1), 0,0, m)$

which is of virtual dimension

$(3 k+1) k-(2 r(3 k, m)-2(2 k+1-m)+m(m-1))=(3 k+1) k-(3 k+1) k=0$.

By Lemma 3.1, this system is non-special, so its actual dimension is also zero. This shows that condition (2) in Theorem 4.3 is fulfilled. The residual system is

$$
\begin{aligned}
\mathcal{L}_{1} & =\mathcal{L}(k-1,1 ; m-1, r(3(k-1)+1, m-1), 0, q(3(k-1)+1, m-1), 0) \\
& =B(k-1,1, m-1)
\end{aligned}
$$

by Lemma 4.5. Note that the subscheme $Z_{1}:=\operatorname{Res}_{Q}\left(Z_{0}^{\prime}\right)$ then satisfies conditions (i)-(iii) of Theorem 4.3 .

Case $B(k, 1, m)$. In this case we use two specializations. First set

$$
Z_{0}^{\prime}=R(1,2 k+1-m, 0,0, m(m-1), 2 k, 0),
$$

resulting in

$$
\mathcal{L}_{1}=\mathcal{L}\left(k-1,2 ; m-1, \frac{3}{2} k^{2}-\frac{5}{2} k+1-\frac{1}{2} m^{2}+\frac{1}{2} m, 2 k, \frac{1}{3} m^{3}-m^{2}+\frac{2}{3} m, 0\right)
$$

and

$$
\Lambda_{1}=\Lambda\left(k, 3 k+1 ; 3 k^{2}-k+2,2 k, 0, m\right),
$$


which system is zero-dimensional. Then we set

$$
Z_{1}^{\prime}=R(0,1,2 k, 0,0,0,0)
$$

and obtain the residual system

$\mathcal{L}_{2}=\mathcal{L}(k-1,0 ; m-1, r(3(k-1), m-1), 0, q(3(k-1), m-1), 0)=B(k-1,0, m-1)$,

and the trace system

$$
\Lambda_{2}=\Lambda\left(k-2,3 k-1 ; 3 k^{2}-3 k-m^{2}+m, 0,2, m-1\right),
$$

with $h^{0}\left(\Lambda_{2}\right)=0$.

Case $B(k, 2, m)$. In this case we use the specialization

$$
Z_{0}^{\prime}=R(1,2 k+2-m, 0,0, k+1+m(m-1), 0,0) .
$$

We obtain

$$
\mathcal{L}_{1}=\mathcal{L}(k, 0 ; m-1, r(3 k, m-1), 0, q(3 k, m-1), 0)=B(k, 0, m-1)
$$

and

$$
\Lambda_{1}=\Lambda\left(k, 3 k+2 ; 3 k^{2}+6 k+3,0,0, m\right)
$$

which is of dimension 0 .

\subsubsection{The injective cases}

With $d=3 k+\varepsilon$, the initial state in every case now is

$$
\mathcal{L}(k, \varepsilon ; m, r(3 k+\varepsilon, m)+1,0,0,0) .
$$

Case $I(k, 0, m)$. We have $\mathcal{L}_{0}=\mathcal{L}(k, 0, m, r(3 k, m)+1,0,0,0)$ so that

$$
\operatorname{vdim}\left(\mathcal{L}_{0}\right)=-3 k-1+\frac{1}{3} m(m-1)(m+1)<0
$$

for $d=3 k \geqslant d_{0}(m)=3\left(\begin{array}{c}m+1 \\ 3\end{array}\right)$.

We apply the specializations

$$
\begin{aligned}
& Z_{0}^{\prime}=R(1,2 k+1-m, 0,0,0,0, m(m-1)-2) \\
& Z_{1}^{\prime}=R\left(1,2 k+1-m-\left(\frac{1}{2} m(m-1)-1\right), 0, \frac{1}{2} m(m-1)-1,0,0,0\right)
\end{aligned}
$$

By Lemma 4.5 the trace systems are

$$
\begin{aligned}
& \Lambda_{1}=\Lambda((3 k, k-1) ; 2(r(3 k, m)+1-(2 k+1-m)), m(m-1)-2,0, m) \\
& \Lambda_{2}=\Lambda\left((3 k-2, k-2) ; 3 k^{2}-3 k+2-2 m^{2}+4 m, 0,0, m-1\right)
\end{aligned}
$$

It is easy to see that both of these have non-positive virtual dimensions for $d \geqslant$ $d_{0}(m)$, and thus actual dimension zero.

Note also that we have the identity

$$
r(3 k, m)+1-(2 k+1-m)-(2 k+1-m)=r(3(k-2)+2, m-2)+1 .
$$

The final residual system thus is

$$
\mathcal{L}_{2}=\mathcal{L}(k-2,2, m-2, r(3(k-2)+2, m-2)+1,0,0,0)=I(k-2,2, m-2) .
$$


Case $I(k, 1, m)$. Here $\mathcal{L}_{0}=\mathcal{L}(k, 1, m, r(3 k+1, m)+1,0,0,0)$, which has virtual dimension

$$
\operatorname{vdim}\left(\mathcal{L}_{0}\right)=-3 k-2+\frac{1}{3} m(m-1)(m+1)<0 .
$$

We apply the specialization

$$
Z_{0}^{\prime}=R(1,2 k+2-m, 0,0,0,0,0)
$$

which by the identity

$$
r(3 k+1, m)+1-(2 k+2-m)=r(3(k-1)+2, m-1)+1
$$

yields

$$
\mathcal{L}_{1}=\mathcal{L}(k-1,2, m-1, r(3(k-1)+2, m-1)+1,0,0,0)=I(k-1,2, m-1)
$$

as the residual system and

$$
\Lambda\left((k-1,3 k+1) ; 3 k^{2}+3 k+2-m^{2}+m, 0,0, m\right)
$$

as the trace system. Its virtual dimension is

$$
\operatorname{vdim}\left(\Lambda_{1}\right)=-k-2+m^{2}-m<0,
$$

so $h^{0}\left(\Lambda_{1}\right)=0$.

Case $I(k, 2, m)$. This is the most difficult case - it requires the use of zig-zags, and the specializations and their number depend on the multiplicity $m$ of the fat line as well as on the divisibility of $m$ by 3 . In this step, additionally, the reduction goes to one of the bijectivity cases.

$$
\begin{aligned}
& \mathcal{L}_{0}=\mathcal{L}(k, 2, m, r(3 k+2, m)+1,0,0,0) \text { and } \\
& \qquad \operatorname{vdim}\left(\mathcal{L}_{0}\right)=-2 k-2+\frac{1}{3} m(m-1)(m+1)<0
\end{aligned}
$$

In each case the first specialization will be

$$
Z_{0}^{\prime}=R(1,2 k+2-m, 0,0,0,0, k+m(m-1)+1) .
$$

Define further for $p=2, \ldots, m-1$

$$
Z_{p-1}^{\prime}=R\left(1,2 k+2-m-\left\lfloor\frac{p-1}{3}\right\rfloor-\left\lfloor\frac{t_{z_{p-1}}+1}{2}\right\rfloor, 0,\left\lfloor\frac{t_{z_{p-1}}+1}{2}\right\rfloor, 0,0, t_{z_{p}}\right),
$$

where

$$
t_{z_{p}}=\left\{\begin{array}{ll}
k+p m(m-p)+\frac{1}{3} p(p-1)(p+1)-2 p+1 & \text { if } p \equiv 1,2 \\
p m(p-m)+\frac{1}{3} p(p-1)(p+1)-2 p+2 \frac{p}{3} & \text { if } p \equiv 0
\end{array} \quad(\bmod 3) .\right.
$$

Note that $t_{z_{p}}$ is chosen in a way that guarantees the corresponding trace systems to have virtual dimension zero, and thus actual dimension zero. 
Subcase $I(k, 2, m=3 \ell)$. In this case we consider the sequence $Z_{0}, Z_{1}, \ldots, Z_{m-2}$ defined above and use as a final step $Z_{m-1}=\operatorname{Res}_{Q}\left(Z^{\prime}\right)$ for

$$
Z^{\prime}=R\left(1,2 k+2-m-(\ell-1)-\left\lfloor\frac{t_{z_{m-2}}+1}{2}\right\rfloor+1,0,\left\lfloor\frac{t_{z_{m-2}}+1}{2}\right\rfloor, 0,0,0\right)
$$

The final residual system is

$$
\mathcal{L}_{m-1}=\mathcal{L}\left(k-2 \ell+1,1,1, r(3 k+2,3 \ell)+\frac{21}{2} \ell-\frac{23}{2} \ell+3-6 k \ell+2 k, 0,0,0\right) .
$$

Since

$$
r(3 k+2,3 \ell)+\frac{21}{2} \ell-\frac{23}{2} \ell+3-6 k \ell+2 k=r(3(k-2 \ell+1)+1,1)
$$

and $q(3(k-2 \ell+1)+1,1)=0$ we have

$$
\mathcal{L}_{m-1}=B(k-2 \ell+1,1,1) .
$$

The final trace system is

$\Lambda_{m-1}=\Lambda\left(k-2 \ell, 3 k-6 \ell+6,2 r(3 k+2,3 \ell)-12 k \ell-16 \ell+21 \ell^{2}+3 k+3-9 \ell^{3}, 0,0,2\right)$,

which has virtual dimension $-2 k-2+\frac{1}{3} m(m-1)(m+1)<0$.

Subcase $I(k, 2, m=3 \ell+1)$. Consider the sequence $Z_{0}, Z_{1}, \ldots, Z_{m-1}$ defined above and use as a final step $Z_{m}=\operatorname{Res}_{Q}\left(Z^{\prime}\right)$ for

$$
Z^{\prime}=R\left(1,2 k+2-m-\ell-\left\lfloor\frac{t_{z_{m-1}}+1}{2}\right\rfloor+1,0,\left\lfloor\frac{t_{z_{m-1}}+1}{2}\right\rfloor, 0,0,0\right)
$$

The final residual system is

$$
\mathcal{L}_{m}=\mathcal{L}\left(k-2 \ell, 0,0, r(3 k+2,3 \ell+1)+\frac{21}{2} \ell^{2}-\frac{1}{2} \ell-1-6 k \ell-2 k, 0,0,0\right)
$$

which thanks to the identities

$$
r(3 k+2,3 \ell+1)+\frac{21}{2} \ell^{2}-\frac{1}{2} \ell-1-6 k \ell-2 k=r(3(k-2 \ell), 0)
$$

and $q(3(k-\ell), 0)=0$ equals the system $B(k-2 \ell, 0,0)$, as required. The final trace system is

$$
\Lambda_{m}=\Lambda\left(k-2 \ell-1,3 k-6 \ell+2,2 r(3 k+2,3 \ell+1)-12 k \ell-4 k-9 \ell^{3}+12 \ell^{2}+\ell-2,0,0,1\right)
$$

Also in this case we have

$$
\operatorname{vdim}\left(\Lambda_{m}\right)=-2 k-2+\frac{1}{3} m(m-1)(m+1)<0 .
$$


Subcase $I(k, 2, m=3 \ell+2)$. Use as in the first subcase the sequence $Z_{0}, Z_{1}, \ldots, Z_{m-2}$ defined above and use as a final step $Z_{m-1}=\operatorname{Res}_{Q}\left(Z^{\prime}\right)$ for

$$
Z^{\prime}=\left(1,2 k+2-m-\ell-\left\lfloor\frac{t_{z_{m-2}}+1}{2}\right\rfloor+1,0,\left\lfloor\frac{t_{z_{m-2}}+1}{2}\right\rfloor, 0,0,0\right)
$$

The final residual system is

$$
\mathcal{L}_{m-1}=\mathcal{L}\left(k-2 \ell, 0,1, r(3 k+2,3 \ell+2)+\frac{21}{2} \ell^{2}+\frac{5}{2} \ell-6 k \ell-2 k, 0,0,0\right)
$$

with

$$
r(3 k+2,3 \ell+2)+\frac{21}{2} \ell^{2}+\frac{5}{2} \ell-6 k \ell-2 k=r(3(k-2 \ell), 1)
$$

and $q(3(k-2 \ell), 1)=0$. The final trace system is

$\Lambda_{m-1}=\Lambda\left(k-2 \ell-1,3 k-6 \ell+2,2 r(3 k+2,3 \ell+2)-12 k \ell-4 k-9 \ell^{3}+3 \ell^{2}-2 \ell, 0,0,2\right)$.

Its dimension is zero since

$$
\operatorname{vdim}\left(\Lambda_{m-1}\right)=-2 k-2+\frac{1}{3} m(m-1)(m+1)<0
$$

\section{Final remarks}

We have developed a software to handle calculations necessary here. The software proved indispensable in order to manipulate sets of data and to discover general patterns leading to suitable specializations. Using this software we were not able to find any systems in the range $d<d_{0}(m)$ for which the maximal rank statement in Theorem 4.1 would fail. We therefore expect that the statement holds in these cases as well:

Conjecture 5.1 (Maximal Rank Conjecture). The restriction maps in Theorem 4.1] have maximal rank for all $d \geqslant 1$.

We hope that with some modifications, the software mentioned above might prove useful in similar situations, in particular might help to advance towards the proof of Aladpoosh's Conjecture. We also expect that our results can be generalized to projective spaces of arbitrary dimension. This is a subject of our current research.

Acknowledgement. This project has been started during the mini-workshop "Arrangements of Subvarieties, and their Applications" held in Mathematischem Forschungsinstitut Oberwolfach February 29 - March 5, 2016. We are grateful to MFO for providing perfect working conditions. Part of the paper has been written up during the visit of the last two authors at KTH. We thank KTH for providing financial support and stimulating working atmosphere. The final reductions have been discovered at the Karma coffee place in Kraków. It is a pleasure to acknowledge support and understanding of the Karma team.

Research of DS was supported by DFG research fellowship SCHM 3223/1-1. Research of TS and JS was partially supported by National Science Centre, Poland, grant 2014/15/B/ST1/02197. 


\section{References}

[1] Aladpoosh, T.: Postulation of general lines and one double line in $\mathbb{P}^{n}$ in view of general lines and one multiple linear space. arXiv: 1606.02974

[2] Aladpoosh, T., Ballico, E.: Postulation of disjoint unions of lines and a multiple point. Rend. Semin. Mat. Univ. Politec. Torino 72 (2014), no. 3-4, 127-145

[3] Ballico, E.: Postulation of Disjoint Unions of Lines and a Multiple Point, II. Mediterr. J. Math. 13 (2016), no. 4, 1449-1463

[4] Carlini, E., Catalisano, M. V., Geramita, A. V.: Bipolynomial Hilbert functions. J. Alg. 324 (2010), 758-781

[5] Carlini, E., Catalisano, M. V., Geramita, A. V.: 3-dimensional sundials. Cent. Eur. J. Math. 9(5) (2011), 949-971

[6] Carlini, E., Catalisano, M. V., Geramita, A. V.: On the Hilbert function of lines union one non-reduced point. Ann. Sc. Norm. Super. Pisa Cl. Sci. (5) Vol. XV (2016), 69-84

[7] Dumnicki M., Harbourne, B., Szemberg T., Tutaj-Gasińska H.: Linear subspaces, symbolic powers and Nagata type conjectures. Adv. Math. 252 (2014), 471-491

[8] Hartshorne, R.: Algebraic geometry. Graduate Texts in Mathematics, No. 52. Springer-Verlag 1977

[9] Hartshorne, R., Hirschowitz, A.: Droites en position générale dans l'espace projectif. Algebraic geometry (La Rábida, 1981), 169-188, Lecture Notes in Math., 961, Springer-Verlag 1982

[10] Lazarsfeld, R.: Positivity in algebraic geometry. Ergebnisse der Mathematik und ihrer Grenzgebiete. 3. Folge. A Series of Modern Surveys in Mathematics 48, 49. Springer-Verlag, Berlin 2004 .

[11] Lenarcik, T.: Linear systems over $\mathbb{P}^{1} \times \mathbb{P}^{1}$ with base points of multiplicity bounded by three. Ann. Polon. Math. 101 (2011), 105-122

Thomas Bauer, Fachbereich Mathematik und Informatik, Philipps-Universität Marburg, Hans-Meerwein-Straße, D-35032 Marburg, Germany

E-mail address: tbauer@mathematik.uni-marburg.de

Sandra Di Rocco, Department of Mathematics, KTH, 10044 Stockholm, Sweden.

E-mail address: dirocco@math.kth.se

David Schmitz, Fachbereich Mathematik und Informatik, Philipps-Universität Marburg, Hans-Meerwein-Straße, D-35032 Marburg, Germany

E-mail address: schmitzd@mathematik.uni-marburg.de

Tomasz Szemberg, Department of Mathematics, Pedagogical University of Cracow, Podchorạżych 2, PL-30-084 Kraków, Poland

E-mail address: tomasz.szemberg@gmail.com

Justyna Szpond, Department of Mathematics, Pedagogical University of Cracow, Podchorążych 2, PL-30-084 Kraków, Poland

E-mail address: szpond@up.krakow.pl 